Research Article

\title{
Applications of Small and Medium Enterprise Management System Using Edge Algorithm
}

\author{
Limei Huang $\mathbb{D}^{1,2}$ \\ ${ }^{1}$ School of Economics and Management, China University of Mining and Technology, Xuzhou 221116, China \\ ${ }^{2}$ School of Service and Development, Zhengzhou Normal University, Zhengzhou 450044, China \\ Correspondence should be addressed to Limei Huang; hlmei012@cumt.edu.cn
}

Received 8 April 2021; Revised 24 May 2021; Accepted 30 May 2021; Published 7 June 2021

Academic Editor: Fazlullah Khan

Copyright $\left({ }_{0} 2021\right.$ Limei Huang. This is an open access article distributed under the Creative Commons Attribution License, which permits unrestricted use, distribution, and reproduction in any medium, provided the original work is properly cited.

\begin{abstract}
The traditional small and medium enterprise management system has a low efficiency of operation and management. A small and medium enterprise management system based on an edge algorithm is designed in this paper to address this problem. In the proposed algorithm, the management system is used for efficient information transmission and sharing. The system consists of a collaborative function layer, data service layer, basic environment layer, and application layer. The application layer realizes the storage and integration of enterprise information data through the regulation of the collaborative management center and realizes the privacy encryption protection of information based on the protection mechanism of edge computing. The information is transmitted to the data layer for classified storage through the network communication of the basic environment layer. The collaborative function layer takes the process management control engine combined with each service interface to complete the information retrieval of the data layer. It uses the algorithm based on the number of fusion common scoring users and information interest relationship to query and push information. The test results show that the system has good information query performance, pushing query information in one minute. The running function can meet the user needs and effectively achieve information privacy protection.
\end{abstract}

\section{Introduction}

A small- and medium-sized enterprise refers to an enterprise with a relatively small scale of personnel and operations. Small- and medium-sized enterprises are an important carrier of mass entrepreneurship and innovation. They play an irreplaceable role in increasing employment, promoting economic growth, scientific and technological innovation, social harmony, and stability. It has important strategic significance for national economic and social development. Different countries, different stages of economic development, and different industries will have certain differences in their definition standards, which will change with the dynamic development of the economy [1]. Countries generally define small- and medium-sized enterprises in terms of quality and quantity. The qualitative indicators mainly include the organizational form, financing mode, and industry status of the enterprise. In contrast, the quantitative indicators mainly include the number of employees, paid-in capital, and total assets. A management system is composed of a management process and method to achieve the goal of an enterprise through the deployment of interrelated management positions and personnel [2]. Enterprise management can adopt the method of systematic analysis and comprehensively study various professional management of enterprises. These combine the internal conditions and external environment of production and operation activities. It also works on quantitative analysis and qualitative analysis. It selects the optimal scheme to improve economic benefits and promote production development [3]. Enterprise management systems perform decisionmaking, planning, organization, leadership, monitoring, analysis, and so forth. They provide real-time, relevant, accurate, and complete data and provide a decision-making basis for managers and information sharing among various enterprise departments. 
Small and medium enterprises management was introduced in China long after its development in other countries. Other countries have received the concept of digital management relatively earlier, for example, extreme programming, Cockburn's crystal series method, opensource, and other technologies. Therefore, the development of foreign enterprise management systems has become increasingly mature. Domestic large-scale enterprises also have a lot of discussions and implementation. However, a few successful developers with advanced large-scale application platforms and some successful developers only use foreign products for integration and transformation [4]. In China, the biggest obstacle to the management of small- and medium-sized enterprises is that the development cost is too high, and the training of users is not enough. As a result, the development of the system is easy to die prematurely, and the system cannot play its benefits. However, the domestic development of small-scale application systems is very successful, such as personnel and financial system, purchase, sales and inventory systems, and production management systems.

Edge computing is an open platform that integrates the core capabilities of the network, computing, storage, and application to provide the nearest end services on the side close to the object or data source. Its application is initiated at the edge side, which produces a faster network service response. It meets the basic needs of enterprises in real-time business, application intelligence, security, and privacy protection. Edge computing is located between the physical entity and the industrial connection or at the top of the physical entity. In contrast, cloud computing can still access the historical data of edge computing [5]. They are combined with the current problems faced by the middle and lower enterprise management to ease the system management. The high integration of capital flow and information flow makes the enterprise decision-makers know the real data of their operation and sharing information. It improves the management efficiency and timely grasp of the partner's information. Therefore, this paper designs a small- and medium-sized enterprise management system based on an edge algorithm. After the system's function is studied, it is used in the actual enterprise management to improve the management efficiency of small- and medium-sized enterprises and enterprise information security.

The rest of the paper is organized in accordance with the following pattern: in Section 2, we discuss the enterprise management system using an edge algorithm; the analysis is performed in Section 3, and Section 4 concludes the paper.

\section{Application of Small and Medium Enterprise Management System Using Edge Algorithm}

In this section, we discuss the system architecture, applications, and collaborative function layers.

2.1. System Architecture. The construction of small and medium enterprise management systems based on edge algorithms needs to be analyzed from technology and management needs. The management needs to understand and use the management characteristics of small- and medium-sized enterprises. From the perspective of collaborative engineering and system engineering applicability of small- and medium-sized enterprises, its functional structure can be appropriately simplified and reorganized. It establishes a collaborative management center with core manufacturing enterprises, including collaborative planning, selection and management matching, information exchange, access control management, data security management, and other management systems [6]. The system can maintain the core idea of enterprise collaborative management and value chain management and enhance the applicability and operability of the integrated platform. The management system should be networked, intelligent, integrated, virtualized, and secure. Therefore, a small- and medium-sized enterprise management system based on an edge algorithm is designed. The system consists of a collaborative function layer, data service layer, basic environment layer, and application layer. Its architecture is shown in Figure 1.

2.1.1. Collaborative Function Layer. This layer is the top layer of the system, and it is also one of the core leading layers of system management. It includes four functional components: enterprise collaborative planning component, selection and matching component (supplier, cooperative plant, customer, etc.), information exchange component, and access control component. It is mainly used to realize information exchange and queries between internal departments of enterprises. The information exchange and query within certain authority between enterprises and suppliers, cooperative plants, customers, and other related parties [7].

2.1.2. Data Service Layer. It is used to store all information of enterprises, and it is also a data information communication interface in a networked manufacturing environment. It includes information interface agents based on common object request broker architecture (CORBA), for example, customer service interface agent, planning interface agent, design interface agent, supply interface agent, production interface agent, inventory interface agent, and so forth. At the same time, the layer also deploys XML-based information interface agents. The information exchange interface agents, which are mainly used for information exchange between enterprises and related parties.

2.1.3. Basic Environment Layer. The primary environment layer is based on a local area network (LAN), a virtual private network (VPN), and Internet/Extranet/Intranet. It adopts networked object distribution technology and realizes collaborative information exchange and integration among members based on XML technology. It adopts CORBA technology to implement the interoperability between heterogeneous systems through object request broker (ORB). 


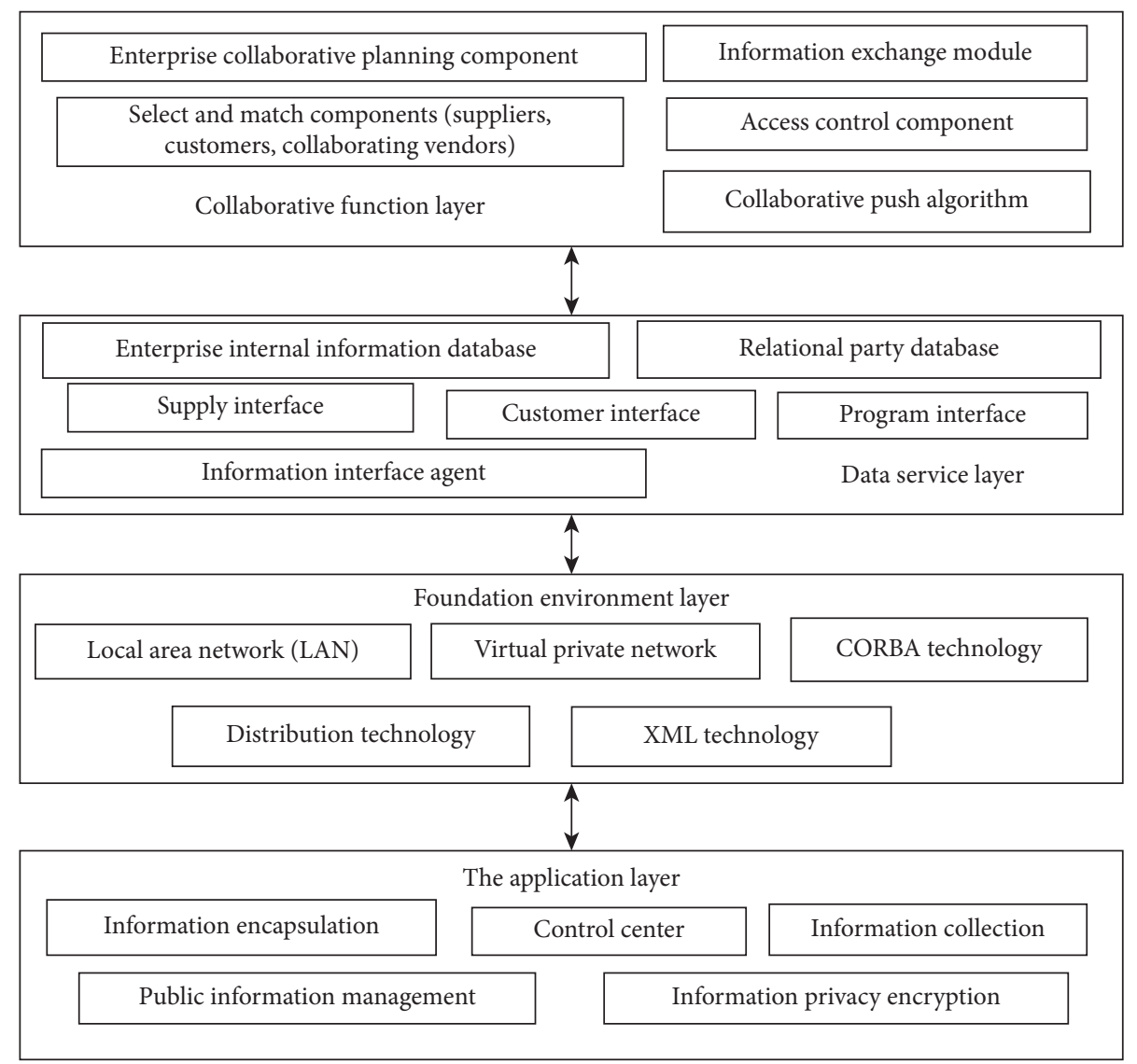

FIgURE 1: System architecture.

2.1.4. Application Layer. The application layer is the bottom layer of the system, and it is also one of the core leading layers of system management. This layer realizes the processing of specific businesses through the control of the collaborative management center. The collaborative management center plays the role of information exchange and system integration center, the manager of public information, and the provider of the interface required for the access, interaction, and interoperability of the member's application system and database [8].

2.2. Application Layer. The following section describes various components of application layers.

2.2.1. Structure of Application Layer. The application layer is one of the core layers of the system, mainly used for the storage and integration of enterprise information data and stores the related information data of related parties, which is the basis of information exchange, query, and access. Therefore, it is imperative to ensure the security of the data in this layer. This layer takes edge computing as the core and sets up a privacy protection mechanism so that when each relational party implements information operation within the scope of authority, it cannot obtain the information of other relational methods [9]. This layer is mainly divided into three parts, namely, client, edge node, and service provider. Its structure is shown in Figure 2.

(1) Client: the main role is to complete the data request and data perception tasks, mainly through the mobile device GPS positioning, various sensors, cameras, and other data acquisition and applications

(2) Edge node: its main function is to complete the whole process of privacy protection through appropriate methods and mechanisms

(3) Service provider: its main function is to provide data aggregation and service request processing, respond to the data processed by edge nodes, and provide feedback results

Assuming that the coverage area of the edge node is $S$, the location privacy protection mechanism $K$ can be generated by adding noise to protect the user's privacy. The main goal is to realize that even if the attacker can know that the user is in a certain area, it cannot determine the real location. When users use location-based services or perform location-related operations, they submit the real location to the edge node and then implement the location privacy protection mechanism at the edge node [10]. Next, the edge node implements a location privacy protection mechanism for the query interest results (or other location-related applications) and filters the useless results according to the real location. 


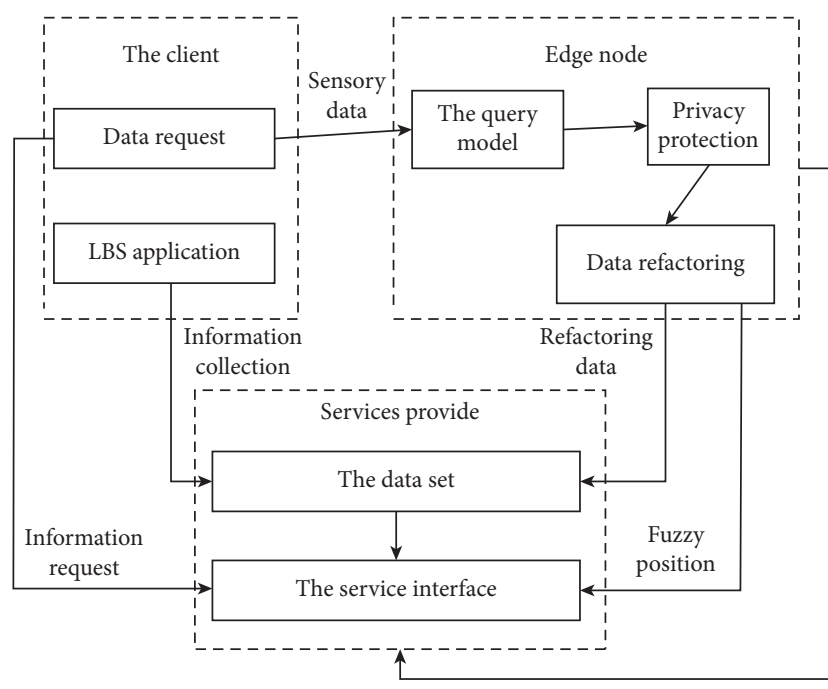

Figure 2: Application layer structure.

\subsubsection{Protection Mechanism Based on Edge Computing.}

The main idea of the geographical indiscernibility mechanism is that the real position is $x_{0}=R$ at any time. However, the reported position is randomly generated according to the noise function $x=R$. The main goal is to ensure that the attacker can only understand the user's area but cannot infer the user's real location from this area. Based on the concept of differential privacy, a formal definition of geographic indiscernibility is given. If the user's real location in the region and any possible location in the region $S$ are represented by $l_{1}$ and $l_{2}$, respectively, then when $K$ only satisfies formulas (1) and (2), it is called geographic indiscernibility mechanism:

$$
\begin{gathered}
P\left[l_{0} \mid l_{1}\right] \leq e^{\varepsilon d\left(l_{1}, l_{2}\right)} P\left[l_{0} \mid l_{2}\right], \\
\forall l_{1}, l_{2}: d\left(l_{1}, l_{2}\right) \leq r .
\end{gathered}
$$

The above definition can be expressed as $P\left(l_{1}\right)(D) \leq e^{\varepsilon d\left(l_{1}, l_{2}\right)} P\left(l_{2}\right)(D), D \in D, D$ representing all data sets and $d\left(l_{1}, l_{2}\right)$ representing the distance between $l_{1}$ and $l_{2}$. The location to submit represents the meaning of the geographic indistinguishability mechanism because two locations $l_{1}, l_{2}$ generate the location $l_{1}, l_{2}$ to be submitted with the same probability. Based on the committed location $l_{0}$, the probability that an attacker can distinguish which is the real location is not negligible [11]. A privacy protection mechanism is proposed to generate the noise location by adding Laplacian distribution noise through the above analysis. When the actual position is $l_{1}$ and $l_{2}$, the probability of a point in a certain region around $l_{0}$ is only one multiplication factor $e^{\varepsilon d\left(l_{1}, l_{2}\right)}$. This property can be achieved by requiring that the probability of generating a point in the nearby region decreases exponentially with the distance from the actual location $l_{1}$. In linear space, Laplace can satisfy this behavior. Laplace distribution with parameter $b$ is recorded as $\operatorname{Lap}(b)$, with density function $P(z \mid b)=(1 / 2 b) \exp (-|z| / b)$ and its variance is $2 b^{2}$. Considering $b=(1 / \varepsilon)$, the density function of the $z$ point can be obtained proportionally. This distribution has the highest density at 0 . For distributions that are not 0 , they can be expressed as $e^{-\varepsilon|z|}$. For the distribution of multidimensional Laplace, it can be proved that $d(x, z)$ is used instead of $|x, z|$.

Considering the parameter $\varepsilon \in R^{+}$and the actual position $l_{1}$, the probability density function of the noise mechanism can be obtained. For any other reporting position $l_{0}$, there is

$$
D_{\varepsilon}\left(l_{1}\right)\left(l_{0}\right)=\frac{\varepsilon^{2}}{2 \pi} e^{-\varepsilon d\left(l_{0}, l_{1}\right)},
$$

where the normalization factor is $\left(\varepsilon^{2} / 2 \pi\right)$ and $\varepsilon$ represents the privacy budget.

The plane coordinate of the probability density function is determined only by distance, so it can be transformed into a coordinate system with the current position $l_{1}\left(x_{1}, y_{1}\right)$ as the origin. The position point $l_{1}\left(x_{1}, y_{1}\right)$ is expressed as a point where the distance from the coordinate point $\theta$ is the angle of the line formed by two points relative to the horizontal axis of the Cartesian coordinate system [12]. According to the standard transformation formula (14), the polar coordinate transformation of Laplacian distribution with the origin as the center is as follows:

$$
D_{\varepsilon}(r, \theta)=\frac{\varepsilon^{2} r}{2 \pi} e^{-\varepsilon r}
$$

where $r$ is the distance from the real position $l_{1}$ to the noisy position $l_{0}$. Because the two random variables representing radius and angle are independent, polar coordinates have a very convenient property. Therefore, the probability density function can be expressed as the product of two sidelines [13]. Then, the formula of two random variables $r, \theta$ is

$$
\begin{aligned}
& D_{\varepsilon, r}=\int_{0}^{2 \pi} D_{\varepsilon}(r, \theta) \mathrm{d} \theta=\varepsilon^{2} r e^{-\varepsilon r}, \\
& D_{\varepsilon, \theta}=\int_{0}^{\infty} D_{\varepsilon}(r, \theta) \mathrm{d} r=\frac{1}{2 \pi} .
\end{aligned}
$$

Therefore, $D_{\varepsilon}(r, \theta)=D_{\varepsilon, r} D_{\varepsilon, \theta}$ is obtained, assuming that the real location point is $l_{1}\left(x_{1}, y_{1}\right)$, then by adding the location point following Laplacian distribution noise, it can be obtained:

$$
l_{0}=\left(x_{1}+r \cos \theta, y+r \sin \theta\right) .
$$

By adding Laplacian noise, location privacy is protected. Because the radius $r$ and the angle are independent in polar coordinates, in order to get the point $(r, \theta)$, it can be obtained from $D_{\varepsilon}(r, \theta)$, respectively.

Because $D_{\varepsilon, \theta}$ is a constant, it is easy to get the value of the random distribution. In this paper, uniform distribution is used, which is randomly generated in the interval.

The polar radius $r$ is obtained by the cumulative distribution function (cdf)C:

$$
C_{\varepsilon}^{-1}=\int_{0}^{r} D_{\varepsilon, r}(\rho) \mathrm{d} \rho=1-(1+\varepsilon r) e^{-\varepsilon r},
$$

where $\rho$ is the threshold value and $C_{\varepsilon}(r)$ represents the radius of random probability point between 0 and $r$. Finally, 
a random number $p$ and the probability interval of uniform distribution $[0,1)$ are generated. If $r=C_{\varepsilon}^{-1}$ is set, it can get the following results:

$$
C_{\varepsilon}^{-1}(p)=\frac{1}{\varepsilon}\left(\frac{p-1}{e}+1\right) .
$$

In this way, the randomness of radius distribution can be realized. In different situations, users have different privacy requirements. For example, when people stay in places they often visit, such as home and office, they want to protect these sensitive places with a higher degree of privacy at the expense of service quality. However, when they are in a public area, it is unreasonable to sacrifice so much quality of service to protect their location privacy [14]. Therefore, a reasonable way is to adjust the privacy level and realize different levels of privacy protection in different scenarios. The privacy level is constrained by $\varepsilon$ obtaining better service quality.

\subsection{Collaborative Function Layer}

2.3.1. Structure of Collaborative Function Layer. In enterprise management, the operation of its business process is not limited to one enterprise, including the joint operation among all enterprises of the related parties. Therefore, collaborative management is the foundation of multiparty cooperation. The technical structure of the collaborative function layer is shown in Figure 3.

Workflow technology will reasonably organize all kinds of information resources inside and outside the enterprise, provide support for collaborative group work, and improve the operation efficiency of the enterprise. Therefore, workflow technology must be used to realize the coordination and monitoring of enterprise business processes. In order to meet the flexibility, availability, scalability, nesting of workflow management, and support for a dynamic application, the collaborative function layer takes the process management control engine to support the collaborative work between enterprise departments. It realizes the full process support for enterprise business process [15], mainly including process model definition, process management control engine, and global task collaborative monitoring service. It can realize the internal personnel information query, cross-department information exchange, cross-enterprise relationship information query, and information push.

\subsubsection{Information Push Based on the Fusion of the Number of} Common Scoring Users and Information Interest Relationship. In the process of related information query operation between various departments and cross-enterprises, the system opens the information operation authority within its authority according to its identity information. In order to ensure that the query personnel can accurately query the information they need, the system can accurately push the information according to the query content. The collaborative function layer combines the common scoring user number and information interest relationship algorithm model. It introduces the knowledge of interest degree and calculates the similarity between information by establishing the interest degree vector between information [16]. The calculation process of the algorithm model can be roughly divided into three processes: (1) establish the user's direct interest in information according to the user's input information content; (2) establish the user's indirect interest in information by combining the direct interest in information and the similarity between users; and (3) fuse the direct interest in information and the indirect interest in information to establish the information interest vector, calculate the similarity between information, and complete information push.

Generally, the user information scoring matrix is a $m \times n$-dimension matrix, which is

$$
R=\left[\begin{array}{lll}
R_{1,1} & R_{1,2} & R_{1, n} \\
R_{2,1} & R_{2,2} & R_{2, n} \\
& & \\
R_{m .1} & R_{m, 2} & R_{m, n}
\end{array}\right],
$$

where the number of users, the number of information, and the score of user $u$ on information $i$ are expressed by $m, n$, and $R_{u, i}$, respectively.

(1) Direct Interest in Information. The user's score of information reflects the user's liking for information. Therefore, it is feasible to use the user's score of information to represent the direct interest of information [17]. If the score of the user $u$ for information $i$ is $R_{u, i}$, the direct interest degree of the user $u$ for information II is as follows:

$$
\operatorname{InD}(u, i)=\frac{R_{u, i}}{R_{\max }},
$$

where the maximum value of the system scoring range is expressed in $R_{\max }$.

(2) Indirect Interest in Information. In collaborative filtering, there is a common problem of the sparsity of scoring data. It is not accurate to measure the similarity between information only by using the direct degree of interest between information. The indirect degree of interest between information also reflects the similarity between information to a certain extent. It can be simply considered that the user's indirect score of information represents the user's indirect interest in information [18]. The indirect score of the user $v$ to information $i$ is defined as follows: if a user $u$ has a rating on information $i$, the user $v$ does not store information $i$, but the user $v$ is similar to the user $u$; then it can be considered that the user $v$ has an indirect score on information $i$ through a user $u$. The direct interest vector $\left\{\mathrm{InD}_{1}, \mathrm{InD}_{2}, \ldots, \mathrm{InD}_{g}\right\}$ of information is set as $i$, where $\{g \leq m, g \in Z\}, u \in\{1,2, \ldots, g\}$, and the similarity between the user $v$ and $u$ is $\operatorname{sim}(v, u)$; then, the indirect interest of the user $v$ to information $i$ is

$$
\operatorname{InD}(u, i)=\frac{\sum_{u=1}^{g} \operatorname{InD}_{u}^{*} \operatorname{sim}(v, u)}{\sum_{u=1}^{g} \operatorname{sim}(v, u)} .
$$

The similarity $\operatorname{sim}(v, u)$ between the user $v$ and $u$ is calculated by cosine similarity. 


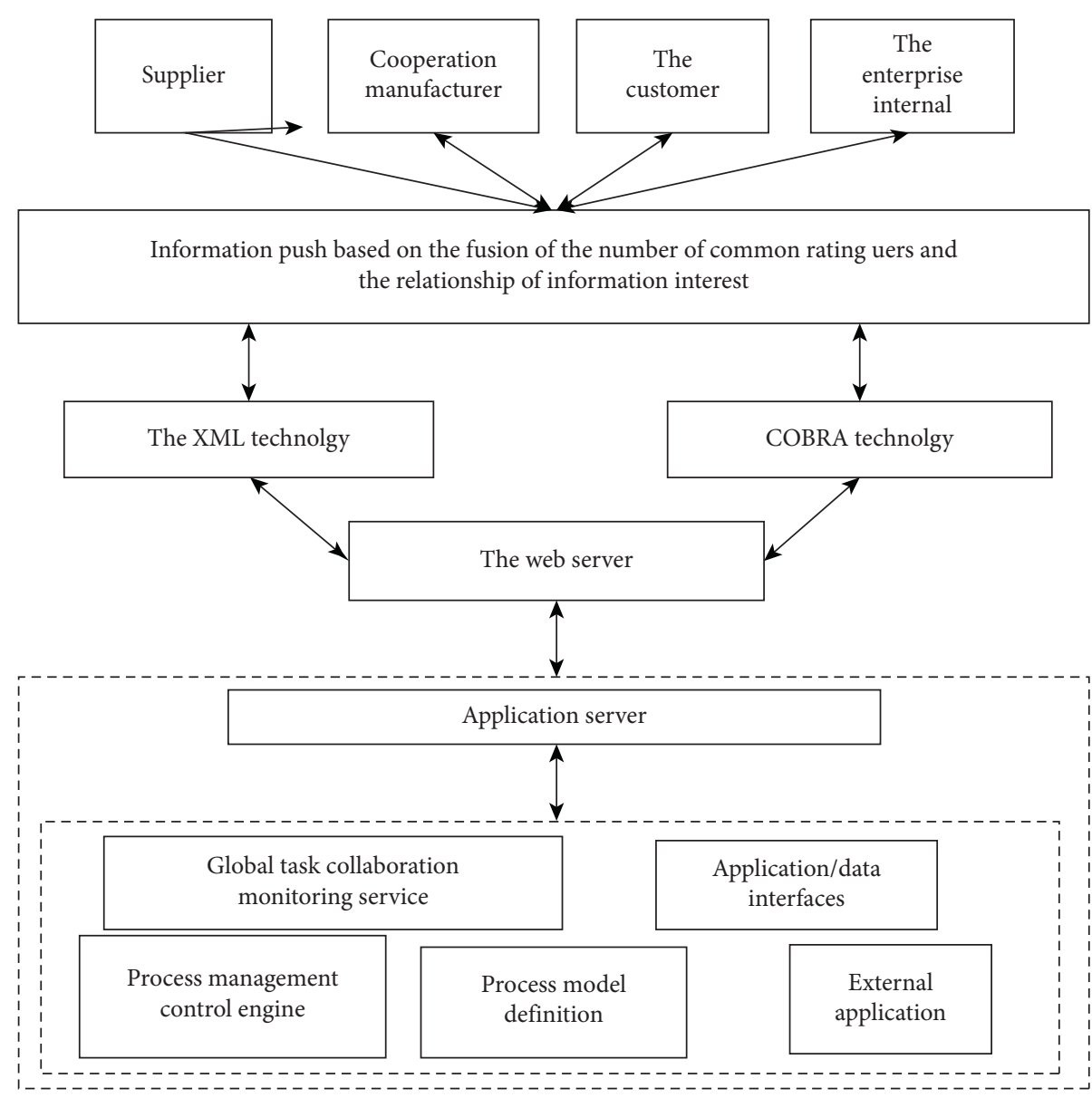

FIgURE 3: Technical structure of synergistic function layer.

(3) Information Similarity. The user's direct interest and indirect interest in information $i$ constitute the information's interest vector In - terest $_{i}=\left\{\operatorname{In}_{1, i}, \operatorname{In}_{2, i}, \ldots, \operatorname{In}_{m, i}\right\}$. If the user $u$ has a direct interest $\operatorname{InD}(u, i)$ in the information $i$, then $\operatorname{In}_{u, i}=\operatorname{InD}(u, i)$; otherwise, $\operatorname{In}_{u, i}=\operatorname{InI}(u, i)$. The information interest relation matrix is composed of information interest vector:

$$
\operatorname{In}=\left[\begin{array}{ccc}
\operatorname{In}_{1,1} & \operatorname{In}_{1,2} & \operatorname{In}_{1, n} \\
\operatorname{In}_{2,1} & \operatorname{In}_{2,2} & \operatorname{In}_{2, n} \\
& & \\
\operatorname{In}_{m, 1} & \operatorname{In}_{m, 2} & \operatorname{In}_{m, n}
\end{array}\right] .
$$

Then, the similarity between information $i$ and information $j$ can be calculated by Pearson similarity:

$$
\operatorname{sim}_{2(i, j)}=\frac{\sum_{u=1}^{m}\left(\operatorname{In}_{u, i}-\overline{\operatorname{In}}_{i}\right)\left(\operatorname{In}_{u, j}-\overline{\operatorname{In}}_{j}\right)}{\sqrt{\sum_{u=1}^{m}\left(\operatorname{In}_{u, i}-\overline{\operatorname{In}}_{i}\right)^{2}} \sqrt{\sum_{u=1}^{m}\left(\operatorname{In}_{u, i}-\overline{\operatorname{In}}_{i}\right)^{2}}}
$$

where $\overline{\operatorname{In}}_{i}$ and $\overline{\operatorname{In}}_{j}$ represent the average interest degree of information $i$ and $j$, respectively.
In order to comprehensively consider the factors that affect the similarity calculation, the similarity between information is calculated from multiple perspectives. Based on the above analysis, this paper proposes a similarity calculation method that combines the number of users and information interest by weighted fitting $\operatorname{sim}_{1}(i, j)$ and $\operatorname{sim}_{2}(i, j)$ :

$$
\operatorname{sim}(i, j)=\lambda \operatorname{sim}_{1}(i, j)+(1-\lambda) \operatorname{sim}_{2}(i, j)
$$

where the weight factor is $\lambda$. In order to better adjust the weight between $\operatorname{sim}_{1}(i, j)$ and $\operatorname{sim}_{2}(i, j)$ and make the similarity calculation results more accurate, the adaptive balance factor model is proposed:

$$
\lambda=\frac{\operatorname{sim}_{1}^{2}(i, j)}{\operatorname{sim}_{1}^{2}(i, j)+\operatorname{sim}_{2}^{2}(i, j)} .
$$

It can be seen that the value range of $\lambda$ is $[0,1]$. When $\lambda$ is $0, \operatorname{sim}_{2}(i, j)$ is used to calculate the similarity between information; when $\lambda$ is $1, \operatorname{sim}_{1}(i, j)$ is used to calculate the similarity between information. Using $\lambda$ weight factors can achieve the effect of dynamic adjustment. 
2.3.3. Overall Implementation Process of the Algorithm. Input: user information rating matrix; output: information recommendation list.

(1) Calculate the similarity between items and the number of users jointly evaluated: according to the user-information scoring matrix, calculate the similarity weight between items and the number of users jointly evaluated. The calculation formulas are as follows:

$$
\begin{aligned}
w(i, j) & =\frac{1}{1+\exp \left(-\left(R_{u, i}-\overline{R_{u}}\right)\left(R_{u, j}-\overline{R_{u}}\right)\right)}, \\
\operatorname{sim}^{\mathrm{jcc}}(i, j) & =\frac{\left|I_{i} \cap I_{j}\right|}{\left|I_{i} \cup I_{j}\right|},
\end{aligned}
$$

where $\operatorname{sim}^{j c c}(i, j)$ value range is $[0,1]$. If the number of scoring users of the two messages is exactly the same, the value is 1 ; if the number of scoring users of the two messages is completely different, the value is 0 .

(2) Calculation of information similarity: according to the similarity of information and the weight of the number of common scoring users, the similarity of information is calculated. The calculation formula is as follows:

$$
\operatorname{sim}_{1}(i, j)=\operatorname{sim}^{\mathrm{jcc}}(i, j)^{*} \operatorname{sim}^{\mathrm{Ad}-\mathrm{pcc}}(i, j)
$$

(3) Calculation of information interest: the user-information scoring matrix is obtained using equations (11) and (12). The user's direct interest and indirect interest in information are calculated, respectively [19]. The interest relation matrix In is established by fusing the information interest.

(4) Calculate information interest similarity: according to the information interest matrix, using equation (15), we calculate the information interest similarity $\operatorname{sim}_{2}(i, j)$.

(5) Fusion information similarity and interest similarity: calculate the adaptive balance factor according to formula (16) and use formula (14) to fuse information similarity and interest similarity $\operatorname{sim}(i, j)$.

(6) Find the nearest neighbor set (NNs) of the target information: according to the Top-n strategy, select the $k$ information with the highest similarity to the target information to form a set [20]. It is regarded as the NNS of the target information.

(7) Scoring prediction: it predicts the user's scoring of target information:

$$
P_{u, i}=\overline{R_{i}}+\frac{\sum_{j \in \mathrm{NNS}_{i}}\left[\operatorname{sim}(i, j)\left(R_{u, j}-\overline{R_{j}}\right)\right]}{\sum_{j \in \mathrm{NNS}_{i}} \operatorname{sim}(i, j)} .
$$

(8) Construction of recommendation list: according to the user's prediction score of target information, select several information with the highest score to form a recommendation column and recommend it to the user.

\section{Results Analysis}

In order to test the management effect and application performance of the system, a medium-sized enterprise in a construction industry is taken as the research object. The enterprise has been established for seven years. It has five departments: the general management department, marketing department, finance department, technology department, and logistics department. There are a total of 246 employees, with annual sales of 38.8 million, and a total of 6 cooperative enterprises. Before the enterprise used the system in this paper, all the information in the enterprise is retrieved and sent by the logistics department.

The experimental environment is a ThinkCentre PC with a 64-bit Windows 7 operating system, 4 -core $3.4 \mathrm{GHz}$ Core i7 processor, and $16 \mathrm{~GB}$ memory. The performance of the system is tested to determine whether the system can meet the needs of users. The test results are shown in Table 1.

According to the test results in Table 1, it can be seen that the system can meet the normal requirements of users: a complete normal login, information input, query, push, privacy protection, information deletion, and information storage functions. It ensures the normal operation of all functions. When the system implements information security and privacy protection, the value of the difference parameter $k$ and privacy parameter $\varepsilon$ in the data set has a certain influence on the security protection. When the two parameters are taken differently, the information security degree is tested. The results are shown in Figures 4 and 5.

According to the test results of Figures 4 and 5, the information security degree also changes with the different values of the parameter $k$. It is showing an irregular floating pattern of up-down-up and down. When the value is 9, the information security degree is the highest; with the increase, the information security degree first increases and decreases. When the value is 11 , the information security degree is the best, so the values of the two parameters are 9 and 11, respectively, and they are used in all subsequent tests.

In order to test the enterprise's information query performance of the system by testing the query selection performance of the system in different quantities, the query selection performance of the system in this paper is shown in Figure 6. It refers to the percentage of all probability numbers meeting the query conditions in the total number. The calculation formula is as follows:

$$
\mathrm{RE}=\frac{\left|Q\left(D_{t}\right)-Q\left(D_{t}^{\prime}\right)\right|}{\max \left(Q\left(D_{t}\right), \gamma\right)},
$$

where the relative error, the result of querying the original data set, and the result of querying the published data set are represented by RE, $Q\left(D_{t}\right)$, and $Q\left(D_{t}^{\prime}\right)$, respectively, and $\gamma$ is the threshold.

According to the test results in Figure 6, the relative error is gradually increasing with the increase of the amount of 
TABLE 1: System function test results.

\begin{tabular}{|c|c|c|}
\hline $\begin{array}{l}\text { Testing } \\
\text { capabilities }\end{array}$ & The test content & $\begin{array}{l}\text { The test } \\
\text { results }\end{array}$ \\
\hline $\begin{array}{l}\text { The login } \\
\text { function }\end{array}$ & $\begin{array}{l}\text { When the user logs in successfully, whether the role of the user can be verified and whether there is a } \\
\text { prompt when the user's name and password are incomplete or incorrect. }\end{array}$ & Yes \\
\hline Input function & $\begin{array}{l}\text { Enterprise employee information, department management information, and other information input } \\
\text { function, whether normal, and input error whether the system will prompt. }\end{array}$ & Yes \\
\hline Query function & $\begin{array}{l}\text { Whether various query conditions can correctly complete the query function, whether the query results } \\
\text { can be displayed correctly, and whether there is a prompt for the data not queried. }\end{array}$ & Yes \\
\hline Push function & After the information query, whether the system can complete the relevant information push. & Yes \\
\hline Privacy features & $\begin{array}{c}\text { Whether the system will prompt and refuse access when the user queries the information that is not } \\
\text { within the authority. }\end{array}$ & Yes \\
\hline Delete function & Whether the function of other modules of the system will be affected after the deletion of data. & No \\
\hline $\begin{array}{l}\text { Storage } \\
\text { capabilities }\end{array}$ & Whether the input information can be completely saved. & Yes \\
\hline
\end{tabular}

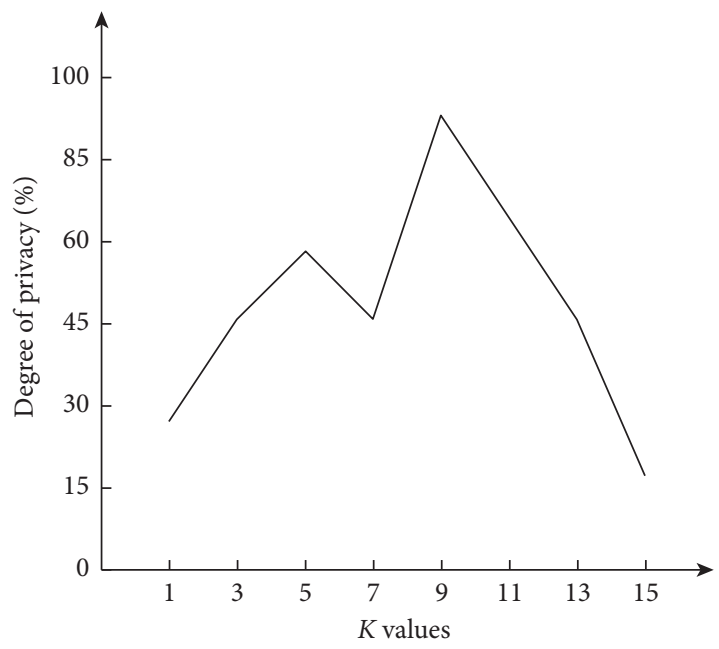

Figure 4: Safety degree test results of different values of $k$.

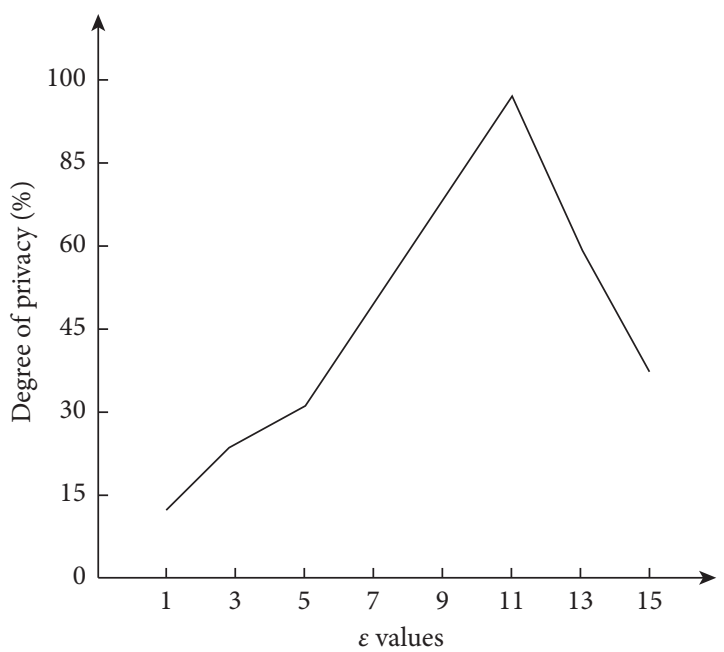

FIGURE 5: Information security degree of different privacy budget values.

query data. However, when the data is $15 \mathrm{~GB}$, the relative error is still less than $0.3 \%$, indicating that the system has good information query performance. It can ensure the user's query demand for the required information. In order to judge the privacy, make statistics of the degree of information privacy of the system when different concurrent users query the same partner information within the same no longer authorized scope. It implements a cross authority query on the partner quotation information of the experimental object in the third quarter of 2019. The results are shown in Figure 7.

According to the test results in Figure 7, with the increase of concurrent users, there is no impact on the privacy and security of the system; the detailed content of quotation information cannot be viewed without authority authentication. The increase of users does not reduce the degree of privacy and security, and the degree of information query is less than $0.06 \%$. It shows that the security and privacy mechanism of the system in this paper has good performance, which can greatly protect the information privacy beyond the scope of the query authority.

After testing the information query, the system will complete the push of relevant query information. The average absolute error widely used in the evaluation of collaborative methods is used to measure the accuracy of the system information push score. The test results are shown in Figure 8 . The average absolute error is calculated as follows:

$$
\text { MAE }=\frac{\sum_{\{u, i\} \in \text { test }} P_{u, i}-R_{u, i}}{N_{\text {test }}},
$$

where the predicted score and real score of user $u$ for information $i$ are, respectively, expressed by $P_{u, i}$ and $R_{u, i}$ and the total score of query information set is expressed by $N_{\text {test }}$. The quality of information push increases with the decrease of MAE value, and the system requirement standard is MAE value within 1 .

According to the test results in Figure 8, with the increase of data, the MAE value of system information push has been floating in the range of $0.29-0.45$, with a small floating degree. When the data volume is $15 \mathrm{~GB}$, the MAE value is 0.38 , which all meet the requirements. The results show that the system can push user information accurately, and the quality of the information is high.

In order to test the application effect of the system in small- and medium-sized enterprises, the efficiency 


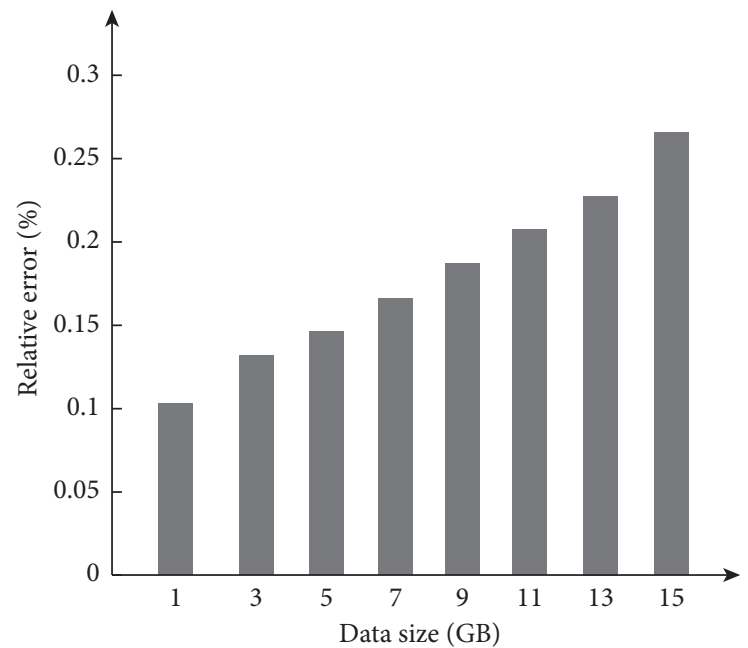

FIgURE 6: Test results of query selection performance of the system.

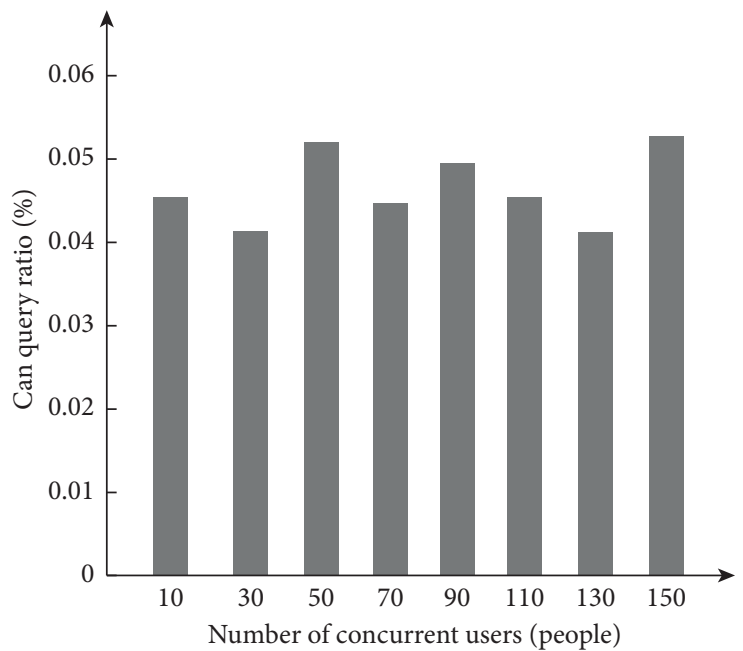

FIgURE 7: Results of privacy degree test.

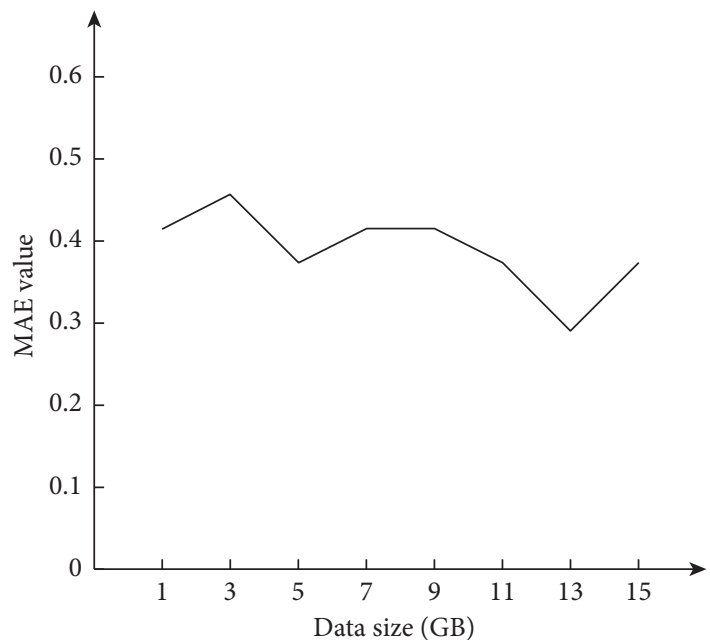

Figure 8: Test results pushed by system information. 


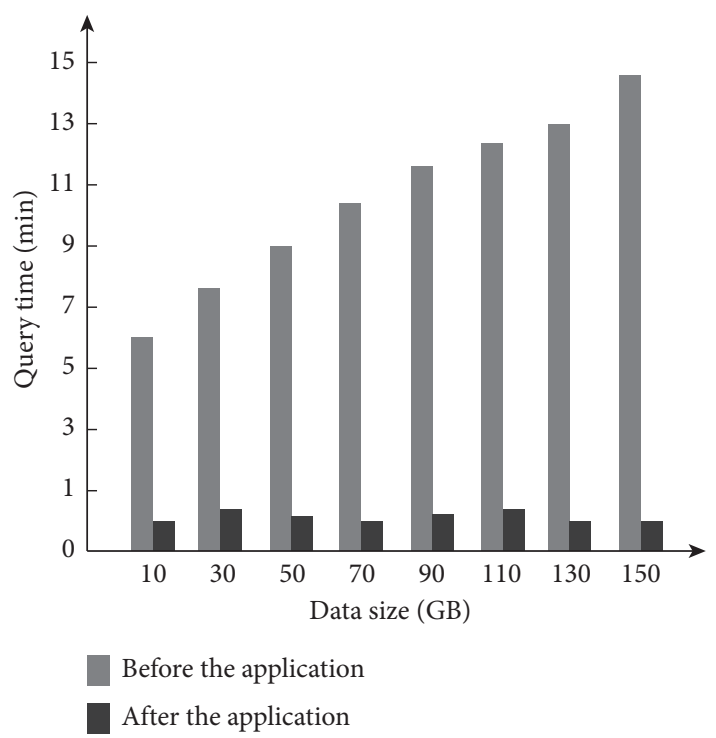

FIgURE 9: Query efficiency test results.

improvement of the partners in the related cooperation, for example, in accounting and other information queries within one year before and after the application of the system is tested. The results are shown in Figure 9.

According to the test results in Figure 9, before applying this system, due to the traditional manual implementation of information retrieval and mail transmission, the efficiency is slow and time-consuming, and the information retrieved may be incomplete. After applying the system in this paper, there is no need for personnel to retrieve and transmit information, whether it is the time information retrieval of various departments within the enterprise of the information viewing of partners. After logging into the system and passing the authentication, the information within the scope of authority can be entirely and timely queried, and the efficiency is significantly improved. When the amount of timely query information is $150 \mathrm{~GB}$, all information can be queried within 1 minute. It shows that the application of the system is high and meets the needs of small- and mediumsized enterprises.

\section{Conclusion}

In the current competitive market, the demand of small- and medium-sized enterprises for collaborative information management is quite different. The purpose of enterprise informatization is to realize the personalization, networking, popularization, digitization, globalization, and marketization of small- and medium-sized enterprises. It aims to promote significantly the development and utilization of information resources and information technology to improve the quality of customer service and social productivity. This research discusses the management and daily operation characteristics of small- and medium-sized enterprises. It takes small- and medium-sized enterprises as an example to realize the purpose of improving the information utilization efficiency and management level, accelerating the speed of information transmission and sharing, and implementing relevant tests. The results show that the proposed system can accelerate information transmission and sharing speed and has a good privacy protection function for the information within the scope of nonauthority.

With the deepening of attention to enterprise applications, our next research task is mainly intelligent enterprise business flow management; that is, when some business flow changes, the program can dynamically reorganize the business flow management part and introduce data warehouse technology and data mining technology into the system to adapt to the continuous improvement of system requirements.

\section{Data Availability}

The data used to support the findings of this study are available from the corresponding author upon request.

\section{Conflicts of Interest}

The author declares no conflicts of interest.

\section{References}

[1] J. Sun, C. Liu, and H. Yuan, "Evaluation of risk management maturity: measurable proactive indicators suitable for Chinese small and medium-sized chemical enterprises," IOP Conference Series: Earth and Environmental Science, vol. 242, no. 4, Article ID 042006, 2019.

[2] G. Kutnjak, D. Miljenović, and A. Mirković, "Improving competitiveness of small and medium-sized enterprises with the application of quality management system," Pomorstvo, vol. 33, no. 1, pp. 11-21, 2019.

[3] K. Meng, G. Lu, and M. Shen, "Research on the problems and countermeasures of salary management in small and medium-sized enterprises," Human Resource Management and Services, vol. 1, no. 1, pp. 16-23, 2019.

[4] S. Lorincová, L. Bajzikova, I. Oborilová, and M. Hitka, "Is corporate culture in small and medium-sized enterprises of forestry and forest-based industry different," Acta Facultatis Xylologiae, vol. 62, no. 2, pp. 121-136, 2020.

[5] D. Sundiman, C. H. Wu, A. Mursidi, S. B. P. Johan, and A. Indahingwati, "Knowledge management key factors: an empirical research on small and medium-sized enterprises in Indonesia," International Journal of Business and Systems Research, vol. 13, no. 2, pp. 139-161, 2019.

[6] E. K. Laitinen and T. Kadak, "Does the company size affect performance management system? pmss in small, mediumsized, and large companies," International Journal of Management and Enterprise Development, vol. 17, no. 3, p. 281, 2018.

[7] V. Varfolomeeva and N. Ivanova, "Investing in small and medium-sized businesses as a factor in the sustainable development of the enterprise," Scientific Research and Development Economics, vol. 8, no. 2, pp. 24-30, 2020.

[8] D. Ufua, R. Osabohien, O. J. Olujobi, D. Imhonopi, and M. E. Isiavwe-Ogbari, "Change management and capacity utilisation: a critical requirement for business sustainability among small and medium-sized enterprises (smes) in Nigeria," International Journal of Innovation Management, vol. 14, no. 10, pp. 438-458, 2020.

[9] S. Adapa and J. Fisher, "Owner-managers' perceptions of corporate social responsibility practices within small and 
medium-sized accounting firms - an Australian study," International Journal of Enterprise Network Management, vol. 11, no. 2, p. 164, 2020.

[10] M. W. Blay, C. S. Cudjoe, and D. O. Okyere, "Corporate compliance with international financial reporting standards: a case study of selected small and medium-sized enterprises (smes) in the cape coast metropolis," International Journal of Engineering Technologies and Management Research, vol. 6, no. 1, pp. 1-12, 2020.

[11] Y. Ma, L. Xia, and Q. Lu, "Research on investment management system and supervision system construction of power grid enterprise group," IOP Conference Series: Earth and Environmental Science, vol. 295, no. 4, Article ID 042083, 2019.

[12] G. Lo, P. D. Santomil, and A. T. Herrera, "The effect of enterprise risk management on the risk and the performance of Spanish listed companies," European Research on Management and Business Economics, vol. 26, no. 3, pp. 111-120, 2020.

[13] M. Jiang, "Research on the financing difficulty of small and medium-sized enterprises under the background of internet based on the perspective of supply chain finance," Finance and Market, vol. 5, no. 2, p. 38, 2020.

[14] W. Sun and Y. Gao, "The design of university physical education management framework based on edge computing and data analysis," Wireless Communications and Mobile Computing, vol. 2021, no. 2, pp. 1-8, 2021.

[15] B. Daniel and A. Sobczyk, "Evaluation of local economic activities as elements of the development of small and medium-sized cities," Zeszyty Naukowe Uniwersytetu Przyrodniczo-Humanistycznego W Siedlcach Seria Administracja I Zarzadzanie, vol. 53, no. 126, pp. 47-54, 2021.

[16] D. Sun, S. Li, W. Yan, F. Jiao, and J. Chen, "Research on user interest expression and recommendation service based on three-dimensional relationship of users and items," International Journal on Recent and Innovation Trends in Computing and Communication, vol. 8, no. 5, pp. 01-15, 2020.

[17] G. Qin, S. Li, and G. Xu, "Research progress on multiscale entropy algorithm and its application in neural signal analysis," Journal of Biomedical Engineering, vol. 37, no. 3, pp. 541-548, 2020.

[18] J. Andreu-Perez, H. Perez-Espinosa, E. Timonet et al., "A generic deep learning based cough analysis system from clinically validated samples for point-of-need covid-19 test and severity levels," IEEE Transactions on Services Computing, vol. 99, p. 1, 2021.

[19] Q. J. Wang and Y. I. Xue, "Research on small and mediumsized enterprise human resources virtualization management," Journal of Central South University of Forestry \& Technology (Social Sciences), vol. 99, no. 3, pp. 39-250, 2010.

[20] A. M. Wang, "Research on stochastic inventory path simulation of E-commerce direct distribution," Computer Simulation, vol. 36, no. 12, pp. 464-467, 2019. 